UNIVERSITY OF GOTHENBURG SCHOOL OF BUSINESS, ECONOMICS AND LAW

WORKING PAPERS IN ECONOMICS

No 522

\title{
How Category Reporting Can \\ Improve Fundraising
}

\author{
Edward Cartwright \\ Amrish Patel
}

December 2011

ISSN 1403-2473 (print)

ISSN 1403-2465 (online)

Department of Economics

School of Business, Economics and Law at University of Gothenburg

Vasagatan 1, PO Box 640, SE 40530 Göteborg, Sweden

+4631786 0000, +46 317861326 (fax)

www.handels.gu.se info@handels.gu.se 


\title{
How Category Reporting Can Improve Fundraising*
}

\author{
Edward Cartwright ${ }^{\dagger}$ and Amrish Patel ${ }^{\ddagger}$ \\ December 9, 2011
}

\begin{abstract}
Many fundraisers report donations using categories such as more than $£ 1000$, more than $£ 10,000$ etc. One naturally wonders how we should categorise donations and whether category reporting can raise more funds than simple uncategorised reporting. To shed light on these questions, we employ a signalling game framework in which both the donor's donation and his benefits of being in a higher category are determined endogenously.

Our analysis suggests that categorised reporting can always improve fundraising. Indeed, we show that both a high and a low category threshold can increase donations. Categorised reporting, especially with a high threshold, can though also lead to the existence of a low donation equilibrium. Fundraisers may then have to choose between: a safer low threshold and a potentially more lucrative high threshold where they would also have to try to coordinate individuals on the desirable equilibrium.
\end{abstract}

Keywords: Fundraising, category reporting, signalling JEL codes: C72, D82, H41

*We thank David Malueg, Georg Kirchsteiger, Martin Dufwenberg, Peter Norman Sorensen, Olof Johansson-Stenman, Johan Stennek, Oleg Shchetinin, Oana Borcan, Jessica Coria and Tobias Dahlström for helpful comments, errors remain our own. Amrish Patel acknowledges the support of a Jan Wallander and Tom Hedelius research fellowship.

†Department of Economics, University of Kent, Canterbury, Kent, United Kingdom, CT2 7NP. E.J.Cartwright@kent.ac.uk

†Department of Economics, University of Gothenburg, Box 640, SE 405 30, Gothenburg, Sweden. Amrish.Patel@economics.gu.se 


\section{Introduction}

Many fundraisers publicise the donations they receive. Although sometimes the exact amounts donated are given, more typically, donations are publicised using categories. To give one of many possible examples: Donors to the Royal Opera House are listed on their website as Platinum, Gold or Silver Patrons depending on whether their donation is more than $£ 21,100$, £9, 050 or $£ 4,950$. Recognising that donors use their donations as signalling devices may explain why fundraisers can use category reporting to influence donations.

Several authors have suggested that donations can be used to signal wealth, altruism, fairness, political leanings or even intelligence (Frank 1985, Glazer and Konrad 1996, Harbaugh 1998a, Bénabou and Tirole 2006, Millet and Dewitte 2007, Ellingsen and Johannesson 2008, Andreoni and Bernheim 2009 and Ellingsen and Johannesson 2011). Supporting these ideas, we certainly observe people donating more when donations are publicly observable (Andreoni and Petrie 2004, Soetevent 2005, Alpizar et al. 2008, Andreoni and Bernheim 2009, Ariely et al. 2009, Carpenter and Myers 2010 and Lacetera and Macis 2010). Given such donor motivations, one would expect categorising donations to change donor behaviour since the "signalling technology" changes. Harbaugh (1998b) reports evidence consistent with this, showing that alumni donations to a law school converged to the category thresholds when category reporting was introduced or changed. For example, a category threshold of $\$ 250$ increased the number donating $\$ 250$ while lowering the number donating $\$ 200$ or $\$ 300$.

Our paper questions whether category reporting can increase total donations. For the law school example, intuition would suggest that category reporting increased the donations of some, those who donated $\$ 250$ rather than $\$ 200$, but decreased the donations of others, those who donated $\$ 250$ rather than $\$ 300$. Should we then expect total donations to be higher or lower? To answer this question we develop a signalling game in which a donor, who can be either 'generous' or 'miserly', donates to a fundraiser. The amount he donates is observed by others who try to infer his generosity, offering him esteem if they believe he is generous. We characterise signalling equilibria which detail expected donations and compare equilibrium donations with exact reporting to those with category reporting to arrive at our results.

We find that category reporting can always improve fundraising. More specifically, we demonstrate that both a high category threshold that increases 
the donation of a generous type and a low category threshold that increases the donation of a miserly type can always be used to increase donations. However, we cannot say that such categorisations will always increase donations if the generous type has little incentive to signal his generosity and/or the miserly type has a strong incentive to match the donation of the generous type. In such instances, the outcome depends on which equilibrium individuals coordinate on. Potentially, category reporting can be ineffective and only likely to decrease donations. Overall, therefore, we show that category reporting can increase or decrease donations depending on the situation. Our results provide several important implications for fundraising policy.

In relating our results to previous literature, it seems most important to mention Harbaugh (1998a), McCardle et al. (2009) and Barbieri and Malueg (2011) who also consider whether category reporting can improve fundraising. These papers assume each donor can get a higher exogenous amount of "prestige" or "selective benefits" from being in a higher category, independently of what other donors $\mathrm{do}^{1}$. Such an assumption is reasonable if donors simply take pride in being in a higher category, or receive consumption goods (Sieg and Zhang 2011), priority ballet tickets for example, from being in a higher category. If however, donors are motivated by signalling and a desire for esteem as we have argued, this assumption seems less tenable. To see why, compare someone donating in a high category where he is the only one donating in this category, to someone donating in a high category when everyone is donating in this category. One would expect that the esteem the donor receives will differ. ${ }^{2}$ Certainly, this appears to be a crucial possibility to consider when comparing category reporting with exact reporting. Unlike previous studies, our signalling game approach allows us to capture and model such a possibility because observers' inferences are endogenous and thus the benefits of being in a higher category are endogenous. Our approach is in line with most recent papers modelling prosocial behaviour and esteem (Bénabou and Tirole 2006, Ellingsen and Johannesson

\footnotetext{
${ }^{1}$ Strictly speaking, strategic concerns are present in Barbieri and Malueg (2011). Since they use a subscription game: a donor only receives the selective benefits if the public good is provided, this depends on others' donations. By contrast, our strategic interaction comes from the selective benefits themselves.

${ }^{2}$ To argue otherwise would require that observers are naïve in some sense in what they infer from the actions of others. We have argued, elsewhere, this may be the case (Cartwright and Patel 2010) but are skeptical it would hold for such 'obvious' distinctions as with category reporting.
} 
2008, Andreoni and Bernheim 2009, Cartwright and Patel 2010, Daughety and Reinganum 2010, Ellingsen and Johannesson 2011 and Soetevent 2011). We shall see that endogenising the benefit of being in a higher category does indeed have important consequences.

Among these consequences are two particularly interesting and new results. The first concerns the efficacy of a low category threshold. A low threshold does lower the donation of a generous type, but in so doing makes it easier for a miserly type to appear generous. The extra donation of the miserly type may be enough to offset the loss from the generous type. Although one might expect this to be true only under some strict conditions, we show that a low threshold can always increase total donations with rather weak assumptions. Furthermore, because category reporting is often most effective when the probability the donor is miserly is relatively high, a low threshold is often more effective because it targets the right type. ${ }^{3}$

Our second novel result arises from the interaction between signalling and categorisation. Categorising donations means unlike most signalling games ${ }^{4}$, our actions and signals are not mapped one-to-one, but many-to-one. This property interferes with the typical "unravelling" of pooling equilibria. For our context, the implication is that a low category pooling equilibrium, where both types only donate what they intrinsically prefer, can exist if donations are categorised. This is an important result as it demonstrates once donor benefits of being in a higher category are endogenised, even if the categorisation is designed optimally, donations may not necessarily increase. Fundraisers should recognise this possibility and act to avoid this outcome.

The rest of the paper is structured as follows. In section 2 we present the model. Section 3 works through the baseline case of exact reporting. Our main results are found in section 4 where we analyse category reporting and compare donations to those with exact reporting. We then conclude in section 5 .

\footnotetext{
${ }^{3}$ Interestingly others have suggested a contribution cap may increase contributions in contests such as in political campaigning or political influence (Che and Gale 1998, Gavious et al. 2002, Drazen et al. 2007 and Baik 2008). In these settings a cap increases the chances of a less endowed individual or group winning, and so increases effort to win. This seems similar in spirit to our finding.

${ }^{4} \mathrm{~A}$ recent notable exception is Harbaugh and To (2008).
} 


\section{$2 \quad$ A signalling model of donations}

Consider a standard signalling game in which there is a sender and a receiver. Interpret the sender as a donor donating to charity or some other organisation, and the receiver as a friend, colleague, or otherwise who observes the donation. The donor is one of two types, $T$, which we call generous $(G)$ and miserly $(M){ }^{5} \quad 6$ The donor's type is private information. The observer's prior is that the donor is generous with probability $p$, for some real number $p \in(0,1)$, and is miserly with probability $1-p .^{7}$

The donor can donate any amount $x \in[0, \infty)$. If he donates $x$ then he receives an intrinsic payoff of $g(x)$ if generous and $m(x)$ if miserly, where $g$ and $m$ are real valued functions. We shall assume that function $g$ is differentiable, achieves a strict maximum at $x_{g}$, is strictly increasing for $x<x_{g}$ and strictly decreasing for $x>x_{g}$. Similarly, we assume that $m$ is differentiable, achieves a strict maximum at $x_{m}$, is strictly increasing for $x<x_{m}$ and strictly decreasing for $x>x_{m}$. The amount $x_{g}$ is thus the intrinsically preferred donation of a generous type and $x_{m}$ that of a miserly type. As one might expect, we assume that the donor intrinsically prefers to donate more if he is generous, $x_{g}>x_{m}$. Finally, we shall assume two single crossing properties,

$$
\begin{array}{ll}
\text { single-crossing property 1: } & g^{\prime}(x)>m^{\prime}(x) \text { for } x \leq x_{m}, \\
\text { single-crossing property 2: } & \left|m^{\prime}(x)\right|>\left|g^{\prime}(x)\right| \text { for } x \geq x_{g} .
\end{array}
$$

The first property implies for all donations $x \leq x_{m}$, a miserly type suffers less intrinsic disutility from reducing his donation by one unit than a generous type does; ruling out counter-signalling equilibria (Feltovich et al. 2002). Analogously, the second property implies the generous type suffers less disutility from increasing his donation by one unit than the miserly type does for $x \geq x_{g}$. This second property will be crucial for many of our results. ${ }^{8}$

\footnotetext{
${ }^{5}$ de Oliveira et al. (2011) have recently identified the existence of "giving types" when it comes to charity.

${ }^{6}$ As we shall see in the following sections we can derive several interesting and transparent results with only two types. Note however, that our results would generalise to finitely many more types.

${ }^{7}$ Note the primary reason for using 'generous' and 'miserly' is the convenience of the letters $g$ and $m$, alternative interpretations are thus easily accommodated.

${ }^{8}$ Technically, we are not going to need some of these assumptions, e.g. we could do without differentiability. But, the assumptions seem mild enough.
} 
Throughout the following it will be useful to have an illustrating example and we shall use a quadratic example where

$$
\begin{aligned}
m(x) & =-(x-a)^{2}, \\
g(x) & =-(x-b)^{2}
\end{aligned}
$$

for some real numbers $a<b$.

Once the donor has made his donation the receiver tries to infer whether or not the donor is generous. At this point we make the key distinction between category reporting and exact reporting. If there is exact reporting the exact amount $x$ donated by the donor is observed. If there is category reporting then there exists a category threshold $\widehat{x}$ such that it is only observed whether the donor donated more than the threshold $x \geq \widehat{x}$ or less $x<\widehat{x}$. ${ }^{9}$ We shall also briefly consider the possibility of no reporting where the receiver is given no information about the donation. The inferences of the receiver will be summarised by an inference function $q$ that maps from observed donation to a probability the donor is generous. That is, $q(x) \in[0,1]$ is the inferred probability that the donor is generous if he donates $x$. Naturally, we shall require in the category reporting case that $q(x)=q\left(x^{\prime}\right)$ if $x, x^{\prime}<\widehat{x}$ or $x, x^{\prime} \geq \widehat{x}$, and that in the no reporting case $q(x)=q\left(x^{\prime}\right)$ for all $x, x^{\prime}$.

If the donor is perceived to be generous then he receives an esteem payoff of $E$, a strictly positive real number. ${ }^{10}$ The total payoff of the donor is his intrinsic payoff plus any esteem payoff, i.e.

$$
\begin{aligned}
U(x, G, q) & \equiv g(x)+E q(x), \\
U(x, M, q) & \equiv m(x)+E q(x) .
\end{aligned}
$$

A pure strategy $s$ details what the donor will donate if generous and if miserly. Thus, $s(T)$ denotes the amount given by the donor if of type $T$.

Note that since our donor cares about the receiver's beliefs, our model is a psychological game (Battigali and Dufwenberg 2009). With regard to signalling games, it is relatively standard with one important exception: When

\footnotetext{
${ }^{9}$ Note that because the donor is one of only two types it is without loss of generality that we assume there is a unique threshold with category reporting. This will become clear as we proceed.

${ }^{10} \mathrm{An}$ equal potential esteem payoff is quite likely for some contexts. For example, an industry's market size is typically well defined and common knowledge, thus the gain from corporate social responsibility activities, such as donating to charity, fits our assumption rather well.
} 
there is category reporting, actions and signals are not mapped one-to-one. Unlike most signalling games (Fudenberg and Tirole 1991, Riley 2001, Sobel 2009), in our model several actions are represented by a single observable signal.

\section{Donations with exact reporting}

We shall start by working through what happens when there is exact reporting and compare this with the case of no reporting. This will provide a benchmark with which to compare donations with category reporting. As is standard in the literature, we shall focus on pure strategy signalling equilibria satisfying the intuitive criterion. ${ }^{11}$ This can be explained as follows.

Given a pure strategy $s$ we shall say that an inference function is consistent with strategy $s$ if the type of the donor is correctly inferred. If there is exact reporting this requires that

$$
q(x)=\left\{\begin{array}{l}
1 \text { if } s(G)=x \text { and } s(M) \neq x, \\
p \text { if } s(G)=x \text { and } s(M)=x, \\
0 \text { if } s(G) \neq x \text { and } s(M)=x
\end{array}\right.
$$

A pure strategy signalling equilibrium consists of a pure strategy $s$ and inference function $q$ such that: (i) The donor maximises his payoff given the inference function $q$. That is,

$$
U(s(T), T, q) \geq U(x, T, q)
$$

for any $x \in[0, \infty)$ and $T=G, M$; and, (ii) The inference function $q$ is consistent with the strategy $s$.

As is standard, the definition of a signalling equilibrium, and condition (1), say nothing about what the inference $q(x)$ should be if $s(G), s(M) \neq x$. This means that there will typically be many signalling equilibria, each with different assumptions on out of equilibrium beliefs. Somehow we need to select among these equilibria and we shall do so by applying the most commonly used refinement for signalling games, the intuitive criterion (Cho and Kreps, 1987). Informally, the intuitive criterion will exclude any signalling

\footnotetext{
${ }^{11} \mathrm{~A}$ complete existence theorem allowing for mixed strategies is available on request. (Editor/referees: See appendix 2)
} 
equilibrium where an out of equilibrium action is attributed to a type who could never have any incentive to undertake that action.

In a standard signalling game, the least cost separating equilibrium, the Riley outcome, is selected by the intuitive criterion (Riley 1979b). We will see this is the case when we have exact reporting in our model. Before coming to this result we need one preliminary. Let,

$$
x_{H}>x_{m} \text { solve } m\left(x_{m}\right)=m\left(x_{H}\right)+E \text {. }
$$

Informally, $x_{H}$ is the most the miserly type would ever donate. The assumptions on $m$ guarantee the existence of a unique value of $x_{H}$.

Proposition 1: If there is exact reporting then there is a unique signalling equilibrium that satisfies the intuitive criterion, described as follows:

Trivially separating. The donor gives $x_{g}$ when generous and $x_{m}$ when miserly if $x_{g} \geq x_{H}$.

Separating. The donor gives $x_{H}$ when generous and $x_{m}$ when miserly if $x_{g}<x_{H}$.

Proof: All proofs are found in the appendix.

The intuition behind this result is as follows: The miserly type has an incentive to donate as much as the generous type, and sacrifice some intrinsic utility for esteem. Single crossing property 2, however, implies that the generous type is willing to donate a sufficiently high amount to deter the miserly type. Thus, the miserly type fails to match successfully the donation of the generous type and donates his intrinsically preferred amount. If the generous type's intrinsically preferred amount is high enough to avoid being matched by the miserly type then he makes that donation (trivial separation); otherwise he increases his donation until he avoids being matched and receives esteem (separation). This is the Riley outcome.

To illustrate we can work through the quadratic example. In this case, $x_{g}=b, x_{m}=a$, and it is simple to work out that $x_{H}=a+\sqrt{E}$. So, applying proposition 1 , we can see that if $b-a \geq \sqrt{E}$ we get a trivially separating equilibrium where the generous type donates $b$ and the miserly type donates $a$. The size of the expected donation is $p b+(1-p) a$. If $b-a \leq \sqrt{E}$ then we 
get a separating equilibrium where the generous type donates $a+\sqrt{E}$ and the miserly type donates $a$. The expected donation is now $a+p \sqrt{E}$. The closer, therefore, is $b$ to $a$ and the larger is $E$ the more the generous type has to sacrifice in order to signal his type.

Interestingly, the donation of neither type depends on $p$. The expected donation however does clearly depend on $p$ and the higher is $p$ then the larger it is. This is captured in the following ratio where we use $E x$ to denote the expectations operator,

$$
\frac{E x[\text { donation } \mid \text { exact reporting }]}{E x[\text { donation } \mid \text { no reporting }]}=1+\frac{p\left(\max \left\{x_{H}, x_{g}\right\}-x_{g}\right)}{x_{m}+p\left(x_{g}-x_{m}\right)} .
$$

Clearly in a trivially separating equilibrium exact reporting has no effect on the expected donation. In a separating equilibrium however, exact reporting increases the expected donation. To illustrate, in the quadratic example one gets

$$
\frac{E x[\text { donation } \mid \text { exact reporting }]}{E x[\text { donation } \mid \text { no reporting }]}=1+\frac{p(a+\sqrt{E}-b)}{a+p(b-a)} .
$$

Donations can, therefore, potentially increase a lot because of signalling if $p$ and $E$ are large. The question we want to ask is whether category reporting can increase donations further.

\section{Donations with category reporting}

Given proposition 1 there are broadly speaking two ways in which one might expect category reporting could increase donations. The first possibility is a high threshold $\widehat{x}>\max \left\{x_{H}, x_{g}\right\}$ that could increase the donation of the generous type. The second possibility is a low threshold $\widehat{x} \leq x_{H}$ that could increase the donation of the miserly type. In examining these possibilities, we again focus on pure strategy signalling equilibria. The only thing we need change in the definition of such an equilibrium is the condition for consistent inferences. The natural requirement, which can be compared to (1), becomes

$$
q(x)=\left\{\begin{array}{l}
1 \text { if } x \geq \widehat{x}, s(G) \geq \widehat{x}, s(M)<\widehat{x} \text { or } x<\widehat{x}, s(G)<\widehat{x}, s(M) \geq \widehat{x} \\
p \text { if } x \geq \widehat{x} \text { and } s(G), s(M) \geq \widehat{x} \text { or } x<\widehat{x} \text { and } s(G), s(M)<\widehat{x} \\
0 \text { if } x \geq \widehat{x}, s(G)<\widehat{x}, s(M) \geq \widehat{x} \text { or } x<\widehat{x}, s(G) \geq \widehat{x}, s(M)<\widehat{x}
\end{array}\right.
$$


Note that if $s(G)=s(M)$ then the definition of a signalling equilibrium again says nothing about what inferences should be if $x<\widehat{x}$ and $s(G) \geq \widehat{x}$ or $x \geq \widehat{x}$ and $s(G)<\widehat{x}$.

\subsection{A high threshold can increase donations}

In Section 3 we found that with exact reporting the generous type donates his intrinsically preferred amount if this is enough to signal his type, or the lowest donation above this amount that does signal his type. Both of these amounts are lower than the maximum he is willing to donate to gain esteem. We define this amount, let

$$
x_{g H}>x_{g} \text { solve } g\left(x_{g}\right)=g\left(x_{g H}\right)+E \text {. }
$$

We can now state the following result.

Proposition 2 (High threshold): For any threshold $\widehat{x} \in\left(\max \left\{x_{g}, x_{H}\right\}, x_{g H}\right]$ there exists a signalling equilibrium satisfying the intuitive criterion where the expected donation is higher than it would be with exact reporting. It can be described as follows:

Separating with high threshold. The donor gives $\widehat{x}$ when generous and $x_{m}$ when miserly.

The expected donation is maximized when $\widehat{x}=x_{g H}$.

By setting a threshold above $\max \left\{x_{g}, x_{H}\right\}$ we make it the case that the only way a generous type can signal his type is by donating more than he would have under exact reporting. If the generous type donates in the high category then we know, using the same reasoning as was relevant for Proposition 1 , the miserly type would never be willing to match his donation. Thus, provided $\widehat{x} \leq x_{g H}$, the generous type has no incentive to deviate from donating $\max \left\{x_{g}, \widehat{x}\right\}$, appearing in the high category, and receiving esteem for signalling his type. The expected donation is higher than with exact reporting. If $\widehat{x}>x_{g H}$, then the intrinsic utility cost of signalling becomes too high for the generous type, so he too would just maximise intrinsic utility, donate $x_{g}$ and appear in the low category. It follows that the equilibrium expected donation is no higher than with exact reporting if $\widehat{x}>x_{g H}$. 
To illustrate, in the quadratic example, we get that $x_{g H}=b+\sqrt{E}$. So, if $b-a \geq \sqrt{E}$, and there would be a trivially separating equilibrium with exact reporting, we get the potential increase in donations of

$$
\frac{E x[\text { donation } \mid \text { high threshold }]}{E x[\text { donation } \mid \text { exact reporting }]}=1+\frac{p \sqrt{E}}{a+p(b-a)} .
$$

If $b-a<\sqrt{E}$ we get the potential increase of

$$
\frac{E x[\text { donation } \mid \text { high threshold }]}{E x[\text { donation } \mid \text { exact reporting }]}=1+\frac{p(b-a)}{a+p \sqrt{E}} .
$$

To put this in some context Figure 1 plots the ratio of expected donations for various parameter values. We see that a well set threshold can, when $a=1$, increase donations by up to a a half. For example, if $b=2$ and $E=1$ the generous type donates 2 with exact reporting and 3 with a high threshold, giving a 33 percent increase in the expected donation if $p=0.5$.

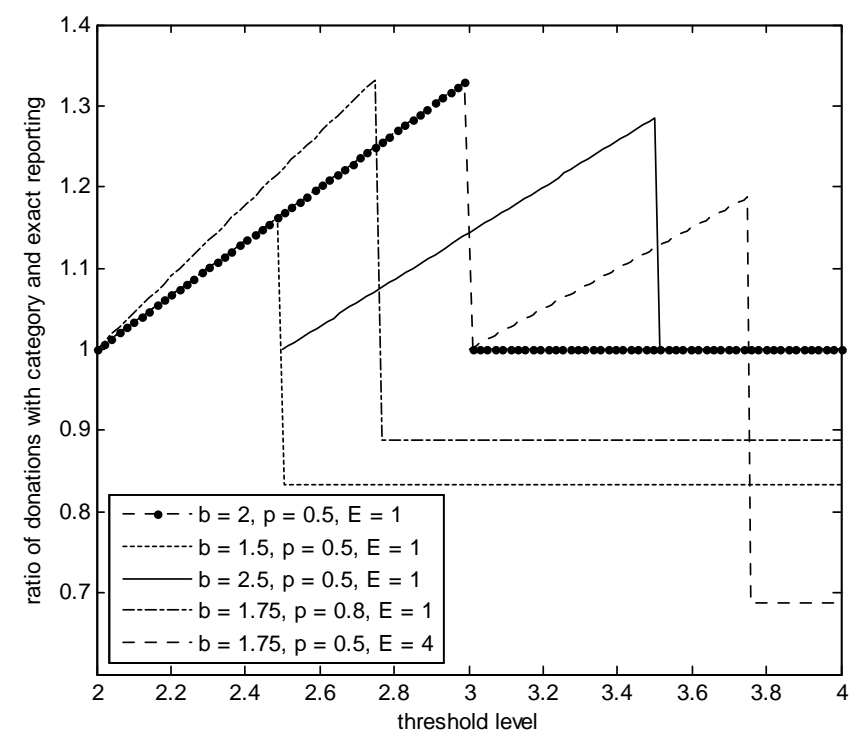

Figure 1: The ratio of donations with category reporting relative to that with exact reporting, when $a=1$.

Overall, therefore, we see that a high threshold can significantly increase donations. It does so by forcing the generous type to donate more in order 
to signal his generosity. It is only if the threshold is pushed too high, so that it is too costly for the generous type to signal his generosity, that a high threshold cannot increase donations.

\subsection{A low threshold can increase donations}

That a high threshold can increase the expected donation is not so surprising. It may be more surprising that a low threshold could increase the expected donation. This is because it is only by lowering the observed donation of the generous type that the miserly type would have any incentive to donate more than $x_{m}$. Thus, to have any effect a low threshold must increase the donation of the miserly type, while potentially lowering the donation of the generous type. These counteracting forces might suggest a low threshold can only work under certain conditions. It turns out, however, that we are able to obtain a definitive result showing that a low threshold can always increase the expected donation. The only thing we require is concavity of the miserly type's intrinsic payoff function.

Since we want the miserly type to end up donating in the same category as the generous type, it will be useful to define the most the miserly type would give in such an instance. Let,

$$
x_{L}>x_{m} \text { solve } m\left(x_{m}\right)=m\left(x_{L}\right)+p E .
$$

In order to be definitive about when a low threshold can increase the expected donation we introduce a further definition. Let

$x_{L L} \in\left[x_{m}, x_{L}\right)$ solve $p \max \left\{x_{g}, x_{L L}\right\}+(1-p) x_{L L}=p \max \left\{x_{g}, x_{H}\right\}+(1-p) x_{m}$.

Note that if $x_{H} \leq x_{g}$, and so there would be a trivially separating equilibrium with exact reporting, then $x_{L L}=x_{m}$. If $x_{H}>x_{g}$ then $x_{L L}>x_{m}$. The following result considers thresholds where $x_{m}<\widehat{x} \leq x_{L}{ }^{12}$

Proposition 3 (Low threshold): For any threshold $\widehat{x} \in\left(x_{L L}, x_{L}\right]$ there exists a signalling equilibrium satisfying the intuitive criterion where the

\footnotetext{
${ }^{12}$ Note that for clarity we omit analysis of intermediate threshold levels, $x_{L}<\widehat{x} \leq$ $\max \left\{x_{g}, x_{H}\right\}$, from the paper (Editor/referees: see Appendix 2). Since a high or low threshold can always give a greater expected donation than an intermediate threshold, we are not particularly interested in this range.
} 
expected donation is higher than it would be with exact reporting. It can be described as follows:

Pooling above the threshold. The donor gives $\max \left\{\widehat{x}, x_{g}\right\}$ when generous and $\widehat{x}$ when miserly.

The expected donation is maximized when $\widehat{x}=x_{L}$.

Suppose we set some low threshold and the generous type donates in the high category, he then clearly donates $\max \left\{x_{g}, \widehat{x}\right\}$. Since we set the threshold sufficiently low, $\widehat{x} \leq x_{L}$, the miserly type is willing to forgo intrinsic utility to appear in the same category as the generous type. So, he donates $\widehat{x}$. Thus, by making it easier for the miserly type to make the same observed donation as the generous type, we induce the miserly type to donate more. If exact reporting gave a trivially separating equilibrium, clearly a low threshold increases the expected donation as the generous type's donation is unchanged and the miserly type donates more. But why does a low threshold also work when exact reporting gives a separating equilibrium? To understand why concavity of $m$ implies the decrease in the generous type's donation is more than offset by the increase in the miserly type's donation, consider a low threshold of $x_{L}$ using Figure 2 .

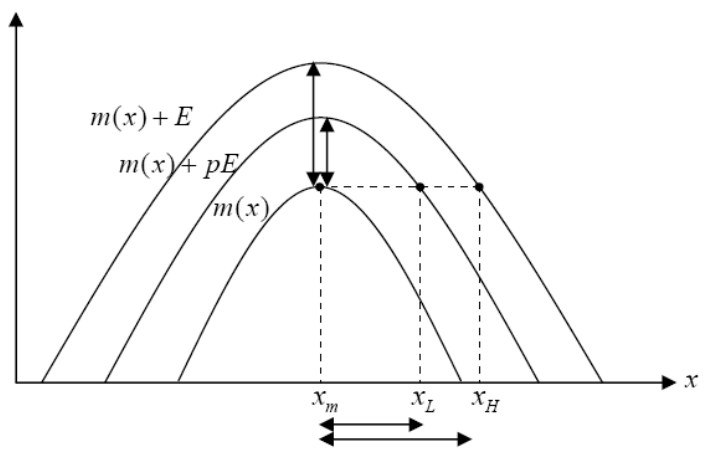

Figure 2: Concavity of $m$ and the efficacy of a low threshold of $x_{L}$

Recall $x_{L}$ and $x_{H}$ are donations where a miserly type is indifferent between donating $x_{m}$ and receiving no esteem or making these larger donations and receiving esteem of $p E$ and $E$ respectively. Concavity of $m$ implies a miserly type gets increasingly more disutility the greater the donation is above $x_{m}$, 
thus a proportional increase in esteem on offer would lead to a less than proportional increase in the indifference donation. As the arrows on the diagram illustrate, this implies that the ratio of esteem on offer is less than the ratio of the increase in donation, $\frac{E}{p E}<\frac{x_{H}-x_{m}}{x_{L}-x_{m}}$ or $x_{L}>p x_{H}+(1-p) x_{m}$. Concavity of $m$ therefore means the expected decrease in donation from a generous type is more than offset by the expected increase in donation from the miserly type, a low threshold works. It does so provided $\widehat{x}>x_{L L}$.

In the quadratic example we get $x_{L}=a+\sqrt{p E}$. So, if $b-a \geq \sqrt{p E}$ (or $\left.x_{L} \leq x_{g}\right)$ we get the potential increase in donations of

$$
\frac{E x[\text { donation } \mid \text { low threshold }]}{E x[\text { donation } \mid \text { exact reporting }]}=1+\frac{(1-p) \sqrt{p E}}{a+p(b-a)} .
$$

If $b-a<\sqrt{p E}$ (or $x_{L} \geq x_{g}$ ) we get the potential increase in donations of

$$
\frac{E x[\text { donation } \mid \text { low threshold }]}{E x[\text { donation } \mid \text { exact reporting }]}=1+\frac{\sqrt{p E}(1-\sqrt{p})}{a+p \sqrt{E}} .
$$

Again, to put this in some context Figure 3 plots the ratio of expected donations for various parameter values. ${ }^{13}$ Significant increases in the expected donation can be observed.

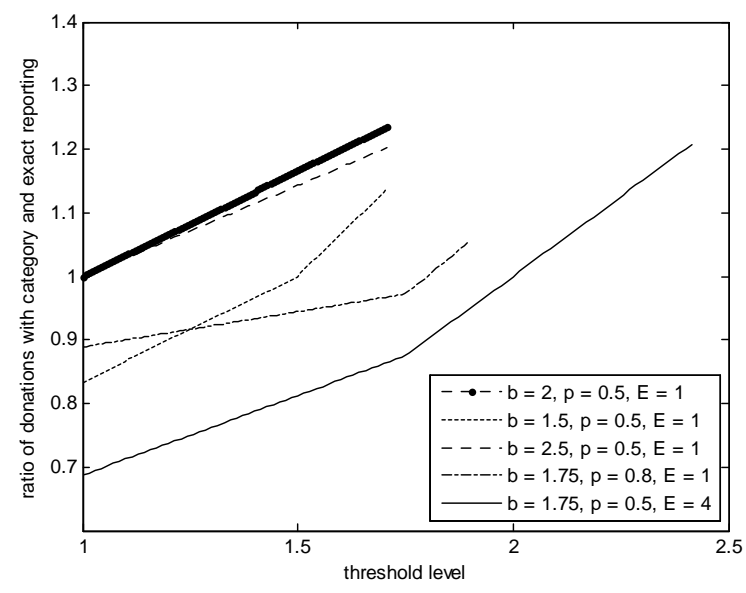

Figure 3: The ratio of donations with category reporting relative to that with exact reporting, when $a=1$.

\footnotetext{
${ }^{13}$ Note that the plots stop at different values of $x$ as $x_{L}$ differs.
} 
That a low threshold can increase donations is an important result because it shows that category reporting can be used effectively in two very different ways. To go one step further we need to question whether the high or the low threshold is most effective.

\subsection{A high or low threshold?}

Given that a high threshold works best when $\widehat{x}=x_{g H}$ and a low threshold works best when $\widehat{x}=x_{L}$, it is a simple matter to compare the expected donation with a low and high threshold and say which is larger. To illustrate we can work through the quadratic example. In this case,

$$
E x[\text { donation } \mid \text { high threshold }]=a+p(b+\sqrt{E}-a)
$$

and

$$
E x[\text { donation } \mid \text { low threshold }]=\left\{\begin{array}{l}
a+\sqrt{p E} \quad \text { if } \sqrt{p E}>b-a \\
(1-p)(a+\sqrt{p E})+p b \text { otherwise }
\end{array} .\right.
$$

So, the low threshold results in a higher expected donation if

$$
\sqrt{p E}>b-a \text { and } p<\frac{E}{(b-a+\sqrt{E})^{2}}
$$

or

$$
\sqrt{p E} \leq b-a \text { and } p<\frac{(\sqrt{5}-1)^{2}}{2} \approx 0.382 .
$$

Figure 4 summarises when the low threshold increases donations the most.

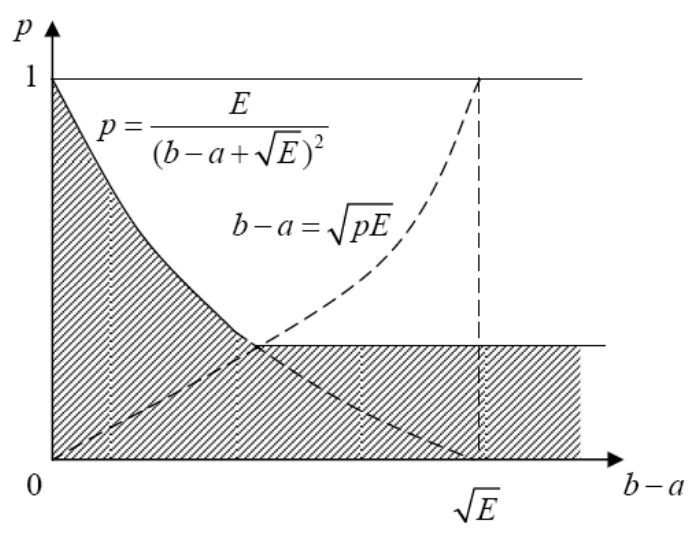


Figure 4: The shaded area is the range of parameter values where a low category threshold increases the expected donation by more than a high threshold.

We see that the low threshold may be best for a non-trivial range of parameters. The lower is $p$, the more likely the donor is miserly, the more likely it is that the low threshold is best.

Figure 5 puts this in context by plotting the expected donation with both a high and low threshold and compares this to the expected donation with exact reporting. The clear picture we get is that category reporting can increase donations significantly and in two very different ways.
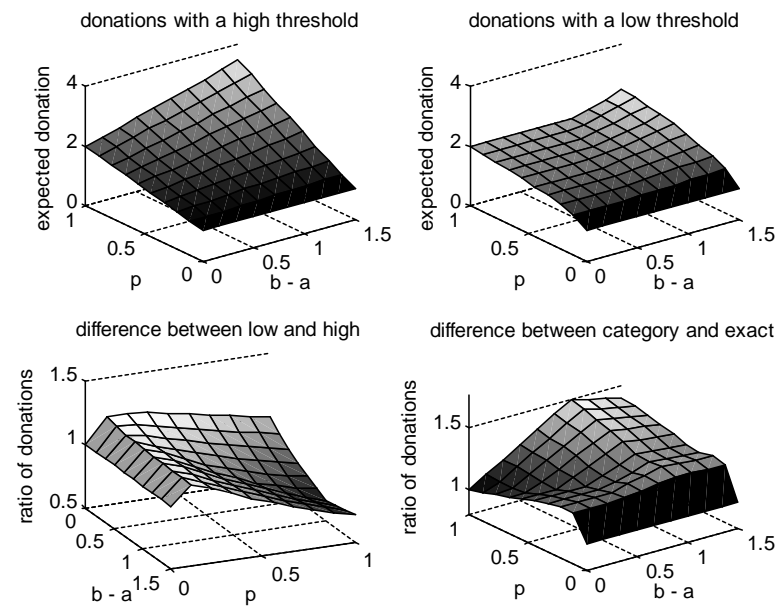

Figure 5: Maximum donations with a high and low threshold, the ratio of maximum donations between a low and high threshold, and the ratio of maximum donations between category and exact reporting, with $E=a=1$.

\subsection{The low category pooling trap}

Our analysis thus far has been relatively optimistic. Not only can category reporting increase the expected donation relative to exact reporting, but it offers a choice of two different methods of doing so. We have however, so far overlooked an important possibility.

While both high and low thresholds can increase the expected donation, we cannot always say they will. This is because there may be a further, 
'less desirable', signalling equilibrium. We define the most the generous type would be willing to donate in order to signal his type rather than pool with the miserly type, let

$$
x_{g L}>x_{g} \text { solve } g\left(x_{g}\right)+p E=g\left(x_{g L}\right)+E .
$$

Proposition 4 (Pooling): For any threshold $\widehat{x} \in\left[x_{g L}, x_{g H}\right]$ there exists a signalling equilibrium satisfying the intuitive criterion where the expected donation is no higher, and potentially lower, than it would be with exact reporting. It can be described as follows:

Pooling below the threshold. The donor gives $x_{g}<\widehat{x}$ when generous and $x_{m}<\widehat{x}$ when miserly.

This result concerns the possibility of a low category pooling equilibrium where both types just donate to maximise their intrinsic utility, $x_{m}$ and $x_{g}$, and both are observed to be in the low category. In other words, category reporting does no better than exact reporting and strictly worse if there was a separating equilibrium with exact reporting.

A low category pooling equilibrium is somewhat surprising. In section 3 we saw that with exact reporting the unique equilibrium sees the generous type increase his donation to a sufficient level to avoid being matched by the miserly type. With category reporting, there exists an equilibrium like this (Proposition 2), but we now see (Proposition 4) that it may not be the only equilibrium. There may also exist an equilibrium where the generous type does not attempt to signal his type. Why does category reporting make this difference? The answer is that category reporting interferes with the standard "unravelling" of pooling equilibria.

To illustrate, suppose that the generous type donates $x_{g}$ and the miserly type matches him by also donating $x_{g}$. With both exact reporting and category reporting, if inferences are consistent, it must be that $q\left(x_{g}\right)=p$. From this one can deduce a value $x_{h} \in\left(x_{g}, x_{H}\right)$ such that the miserly type could not have an incentive to donate more than $x_{h}$. That is, he would be indifferent between donating donate $x_{g}$ or $x_{h}$ if $q\left(x_{h}\right)=1$. Single crossing property 2 implies that the generous type would strictly prefer donating $x_{h}$ or more if $q(x)=1$ for all $x \geq x_{h}$. If there is exact reporting this is enough to mean the 'candidate' pooling equilibrium unravels: The intuitive criterion would impose $q(x)=1$ for all $x \geq x_{h}$ and the generous type would donate $x_{h}$ rather 
than $x_{g}$. If there is category reporting and a threshold $\widehat{x}>x_{g L}$ no such unravelling occurs because, by definition, the generous type prefers donating $x_{g}$ and getting esteem $p E$ rather than donating $\widehat{x}$ and getting esteem $E$. Note that this implies $x_{g L}>x_{h}$ where $x_{h}$ is as just defined.

The existence of this low category pooling equilibrium is important for two reasons. Firstly, it illustrates a more general point on how categorising groups of actions into single signals interferes with unravelling results found in signalling games. Secondly, it calls into question the ability of category reporting to increase donations since donations can never be higher than exact reporting if there is pooling below the threshold. Two obvious questions now arise: Can fundraisers do anything to avoid this situation? And to what extent is the usefulness of category reporting constrained by the existence of this equilibrium?

\subsection{Avoiding low category pooling}

We have now considered the three possible equilibrium outcomes with category reporting. The following result summarises our findings and also clarifies that the equilibria characterised in Propositions 2, 3 and 4 are the only possible pure strategy equilibria satisfying the intuitive criterion.

Corollary 1: Given a threshold $\widehat{x}$ the only pure strategy signalling equilibria satisfying the intuitive criterion ${ }^{14}$ can be summarised as:

(i) If $\widehat{x} \in\left(\max \left\{x_{g}, x_{H}\right\}, x_{g H}\right]$ there exists a separating equilibrium with a high threshold; the expected donation is higher than with exact reporting.

(ii) If $\widehat{x} \in\left(x_{L L}, x_{L}\right]$ there exists an equilibrium with pooling above the threshold; the expected donation is higher than with exact reporting.

(iii) If $\widehat{x} \geq x_{g L}$ there exists an equilibrium with pooling below the threshold; the expected donation is no higher and potentially lower than with exact reporting.

Clearly a fundraiser would prefer to avoid pooling below the threshold. Plausibly, however, depending on $m$ and $g$, the value of $x_{g L}$ could lie anywhere from above $\max \left\{x_{g}, x_{H}\right\}$ to below $x_{L L}$. Thus, multiple equilibria are

\footnotetext{
${ }^{14}$ Strictly speaking, the stronger D1 criterion may be required to rule out other equilibria when $\widehat{x} \in\left(x_{L L}, x_{L}\right]$, in specific cases. [Editor/referees: see appendix 2].
} 
possible. This leaves two options for the fundraiser. They could take steps to coordinate behaviour on the equilibria with higher expected donations. Alternatively, they may restrict themselves to using thresholds $\widehat{x}<x_{g L}$ in order to rule out the possibility of an equilibrium with pooling below the threshold. In this case there will exist a unique signalling equilibrium, meaning we can unambiguously say whether category reporting will increase or decrease the expected donation. The following result summarises when this is possible.

Corollary 2: If $x_{g L}>x_{H}$ then the expected donation can be increased by category reporting with either a high or low category threshold. If $x_{g L}>x_{L L}$ then expected donations can be increased with a low category threshold.

Proof: The only thing of note is that the requirement $x_{g L}>\max \left\{x_{g}, x_{H}\right\}$ can be simplified to $x_{g L}>x_{H}$ because $x_{g L}>x_{g}$.

In general, we know that $x_{L L}=x_{L}<x_{g L}$ if $p$ and $E$ are sufficiently low. It is, thus, only for high $p$ or $E$ that category reporting will not unambiguously increase the expected donation. Intuitively, the large amount of esteem on offer ensures the miserly type is willing to donate more than his intrinsically preferred amount to gain esteem, giving a relatively large expected donation with exact reporting. The high prior probability of the donor being generous means that with category reporting, the generous type has little incentive to signal his type since he receives considerable esteem even if he pools with the miserly type.

To get some sense of what a sufficiently high $p$ or $E$ mean, we return to the quadratic example. Note that $x_{g L}=b+\sqrt{(1-p) E}$. Recall that $x_{H}=a+\sqrt{E}$. Thus, $x_{g L}>x_{H}$ if and only if

$$
b-a>\sqrt{E}(1-\sqrt{1-p}) .
$$

Finding when $x_{g L}>x_{L L}$ is a little bit more cumbersome, but we can begin by noting that

$$
x_{L L}=\left\{\begin{array}{ll}
a & \text { if } b-a \geq \sqrt{E} \\
\frac{p \sqrt{E}+a-p b}{(1-p)} & \text { if } b-a \in(p \sqrt{E}, \sqrt{E}) \\
p \sqrt{E}+a & \text { if } b-a \leq p \sqrt{E}
\end{array} .\right.
$$


It is easily checked that $x_{g L}>x_{L L}$ if $b-a \geq p \sqrt{E}$. From this one can deduce that $x_{g L}>x_{L L}$ if and only if

$$
b-a>\sqrt{E}(p-\sqrt{1-p}) .
$$

Figure 6 illustrates for what values of $p$ and $E$ a high and low threshold can unambiguously be used to increase the expected donation. Figure 7 plots the ratio of the expected donation from exact reporting relative to that from category reporting where the threshold is set to avoid the possibility of pooling below the threshold.

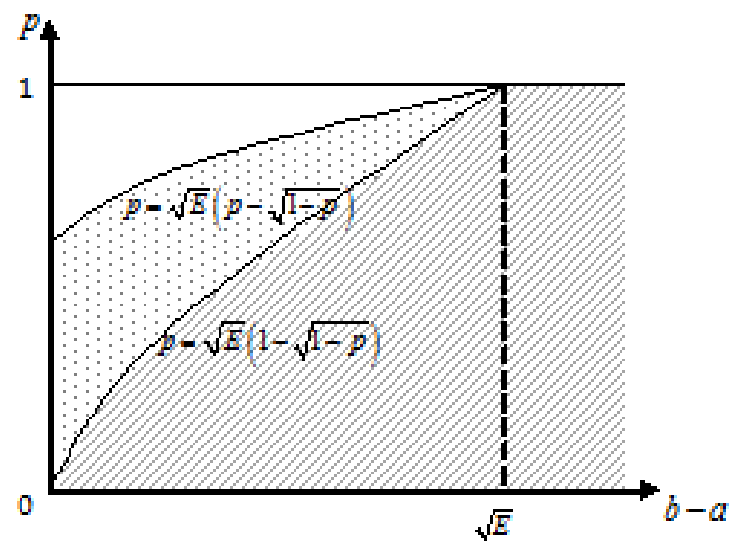

Figure 6: The shaded area is the range of parameters for which a low or high category threshold can unambiguously increase the expected donation.
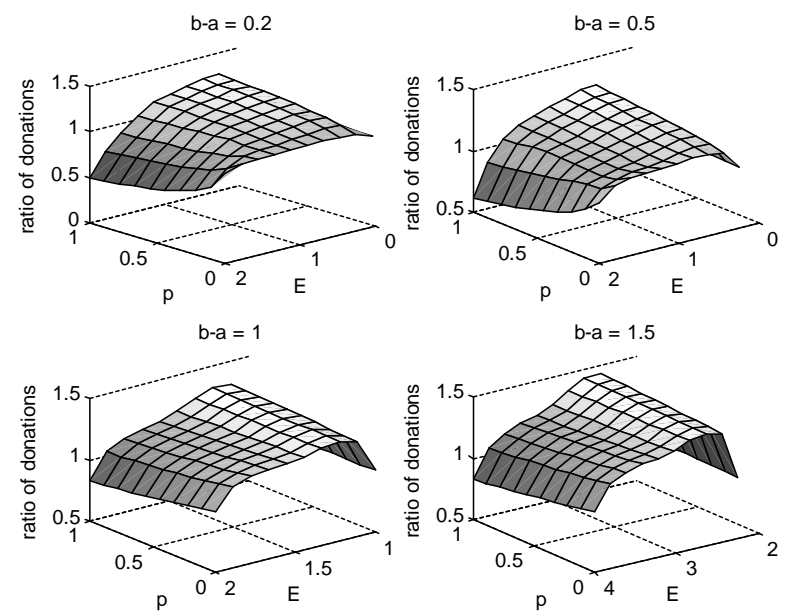
Figure 7: The ratio of maximum donation with category reporting avoiding low category pooling to exact reporting donation, when $a=1$.

We see that for low $p$ and $E$ the ratio is always greater than one, thus category reporting does better than exact reporting. However for higher values of $p$ and $E$, this is not the case. At what point category reporting becomes ineffective depends on the difference between the two types' preferences. The greater the difference, the larger the threshold values of $p$ and $E$ beyond which point category reporting fails.

The last two subsections have added an important qualifier to our view on category reporting, although it can always be used to increase donations one may not have the ability to do so with certainty if $p$ and $E$ are large enough.

\section{Conclusion}

The objective of this paper was to consider whether category reporting of donations can improve fundraising. To address this question we developed a signalling game in which a donor, who was either generous or miserly, makes a donation to a fundraiser; the fundraiser then reports the donation to others who try to infer the donor's type and offer esteem. We found that category reporting can always increase donations. Indeed, both a high and a low category threshold can always do better than exact reporting. However, we also found that when the generous type has insufficient incentive to signal his generosity and/or the miserly type has sufficient incentive to match his donation, a low donation equilibrium also exists. Furthermore, since this equilibrium exists more often when using a high rather than a low category threshold, fundraisers may have to choose between a safer low threshold and a potentially more lucrative high threshold where they also need to coordinate individuals. Context and institutions, therefore, matter a lot.

If fundraisers opt for the safer 'low threshold' that avoids the possibility of multiple equilibria then they almost certainly do so at a cost in terms of the expected donation. Perhaps, therefore, fundraisers should recognise the possibility of multiple equilibria and take steps to coordinate behaviour and expectations on more desirable outcomes. For example, charities often announce early and large donations (e.g. List and Lucking-Reiley 2002). 
With category reporting, this may be one way to coordinate beliefs on the more desirable equilibrium.

In discussing what to learn from our results it is important to compare them to Harbaugh (1998a) and McCardle et al (2009). Both of these studies modelled category reporting assuming (i) a strictly increasing relation between donations and esteem, and that (ii) category reporting does not change the relation between observed donations and esteem. With these two assumptions they show that under very general conditions category reporting can increase donations. In our framework we endogenise the esteem a donor receives by making inferences consistent with donor behaviour. This means that neither assumptions (i) or (ii) need hold. The two consequences of this, which distinguish our results, are that (a) category reporting may easily decrease donations, and (b) it may be appropriate to purposely lower the donation of one type to increase the donation of another.

To explain this distinction further we note that if types separate then our analysis is similar to that of both Harbaugh (1998a) and McCardle et al (2009). This is because when types separate category thresholds can be set in a way to target the donation of one type without affecting the donation of another. If types pool then things are different. Pooling means that a miserly type will copy the donation of a generous type, and observers know this, so assumption (i) no longer holds. Furthermore, category reporting is likely to lead to pooling, meaning that assumption (ii) no longer holds. This latter point means that donations can be very sensitive to category reporting. This is why (a) category reporting may decrease donations and care is needed to set the optimal threshold. Indeed, that donations may increase most if types do pool is why we find (b) that it may be appropriate to lower the observed donation of the generous type.

Useful comparisons can also made between our results and those of Barbieri and Malueg (2011). Although their approach is rather different from ours, a subscription game not a signalling game, one can think of their analysis as examining what happens when different types pool. They suppose fundraisers force donors to choose between some specific donation amounts, thus some donors increase their donation and others decrease it. One of their main results is that if the cumulative density function of donor's intrinsic values is convex then category reporting raises more than exact reporting. We can think of convexity as analogous to having a high probability of a generous type in our model. In our analysis, a high probability of a generous donor meant the existence of the low category pooling equilibrium, in which 
case category reporting may do worse than exact reporting. Intuitively, their model captures the idea that more generous people means more people will increase their donation than decrease it. However, since the benefit of being in a high category is exogenous, their model misses the possibility that the opposite could happen as generous people now have less incentive to increase their donation.

By comparison to previous work on the topic our framework draws out the distinction between pooling and separating. The likely success of, and appropriate form of, category reporting will be different depending on whether there is likely to be pooling or separation of types. This is something we would argue will generally emerge in a signalling model of donations. In particular, our framework was very general with one exception, an assumption of two types. It is clear, however, that our results can be generalised to an arbitrary, finite number of donor types. ${ }^{15}$ While factors such as competing fundraisers and crowding out of intrinsic motivation may attenuate the efficacy of reporting donations, we would still expect the important contrast between pooling and separating to remain; it is not an artefact of our model but something to be expected of signalling equilibria.

Another interesting, and quite general result we expect, is the effect of the signalling technology we analysed. Our signalling model has an interesting property in that actions do not map to signals one-to-one, but many-toone. We found that this property meant that some pooling equilibria do not unravel as they would in standard signalling games and are robust to standard refinements. It is worth noting that in the context of a disclosure game Harbaugh and To (2008) also found this result. Taken together, one may expect this to be a more general result: When actions and signals are mapped many-to-one, pooling equilibria are not broken quite so easily.

When there are multiple equilibria one wonders whether we should expect society to coordinate on pooling or separating. This is an empirical question that we shall not attempt to address here, but one might suggest that if fund-raisers do use category reporting then they must think it works. It is interesting, however, to note the diverse ways in which category reporting is used. For example, the University of Warwick annually sends its alumni

\footnotetext{
${ }^{15}$ Adding types significantly complicates the analysis because the possible permutations of separating, pooling and hybrid equilibria grow exponentially. Propositions 2-4, and their proofs, could, however, easily extend to an arbitrary number of types. Of course, the more types there are the less likely we might think it would be to get the polar extremes where all types pool or all types separate.
} 
a brochure containing a list of donors split into those who donated more or less than $£ 1000$. In this example there is only one category threshold. As a second example, the University of Glasgow's donor wall has six category thresholds ranging from $£ 250$ to $£ 10000$. The more categories there are the more closely is approximated exact reporting of donations. Indeed, given that there may be ethical or practical difficulties in publishing the exact donation of all donors it could be argued that many categories is as close to exact reporting as it is possible to get. Also, charities do express concern about artificial donating thresholds, such as a million pounds, and look for ways to break down such thresholds (Breeze 2009). It is, therefore, not clear whether and when charities do prefer category to exact reporting.

\section{Appendix 1: Proofs of propositions}

\section{Proposition 1 (Exact reporting)}

Given the restriction to pure strategy signalling equilibria there are only two possibilities to consider, (a) a pooling equilibrium where $s(M)=s(G)$, and (b) a separating equilibrium where $s(G) \neq s(M)$. We consider each possibility in turn. Let $x_{Z}<x_{m}$ solve $m\left(x_{m}\right)=m\left(x_{Z}\right)+E$.

Consider a candidate pooling equilibrium where $s(M)=s(G)=\bar{x}$ for some $\bar{x} \in\left(x_{Z}, x_{H}\right)$. Let $x_{h}>x_{m}$ be such that $m(\bar{x})+p E=m\left(x_{h}\right)+E$. The intuitive criterion requires that $q\left(x_{h}+\varepsilon\right)=1$ for some tiny $\varepsilon$. Single-crossing properties 1 and 2 imply $g(\bar{x})+p E<g\left(x_{h}\right)+E$. Thus, a generous type prefers donating $x_{h}+\varepsilon$ to $\bar{x}$. A contradiction.

Consider next a candidate separating equilibrium where $s(G)=\bar{x}$ and $s(M)=\underline{x}$. Consistency of inferences would require $q(\bar{x})=1$ and $q(\underline{x})=0$. This must imply that $\underline{x}=x_{m}$ and either $\bar{x} \leq x_{Z}$ or $\bar{x} \geq x_{H}$, otherwise the miserly type could profitably deviate from the equilibrium. A miserly type weakly prefers $x_{m}$ to $x_{Z}$ or anything less, thus a generous type strictly prefers $x_{g}$ to $x_{Z}$ or anything less (single-crossing property 1), ruling out $\bar{x} \leq x_{Z}$. Furthermore, a miserly type is indifferent between $x_{H}$ and $x_{m}$, thus a generous type strictly prefers $x_{H}$ to $x_{g}$ if $x_{H}>x_{g}$ (single-crossing property $2)$. Finally, inferences $q(x)=0$ for all $x \in\left(x_{Z}, x_{H}\right)$ satisfy the intuitive criterion and guarantee neither type would deviate to such $x$. It follows that there exists a signalling equilibrium satisfying the intuitive criterion as stated in the proposition.

It remains to show uniqueness. The only remaining possibility, however, 
is that $\bar{x}>\max \left\{x_{g}, x_{H}\right\}$ and $q(x)=0$ for all $x<\bar{x}$. This can be consistent with signalling equilibrium, but the requirement that $q(x)=0$ for $x \in\left(\max \left\{x_{g}, x_{H}\right\}, \bar{x}\right)$ violates the intuitive criterion.

\section{Proposition 2 (High threshold)}

Note that single-crossing property 2 implies $x_{g H}>x_{H}$. Fix $\widehat{x}$ such that $\max \left\{x_{g}, x_{H}\right\}<\widehat{x} \leq x_{g H}$ and let $s(M)=x_{m}$ and $s(G)=\widehat{x}$. Equilibrium requires $q(x)=0$ for all $x<\widehat{x}$ and $q(\widehat{x})=1$ for all $x \geq \widehat{x}$. The miserly type has no incentive to deviate since $\widehat{x}>x_{H}$ and the generous type has no incentive to deviate since $\widehat{x} \leq x_{g H}$, therefore we have an equilibrium. Note that, because $\widehat{x}>\max \left\{x_{g}, x_{H}\right\}$, expected donations are higher than with exact reporting, clearly highest when $\widehat{x}=x_{g H}$. Next consider $\widehat{x}>$ $x_{g H}$. Note that $s(G)=\widehat{x}$ is no longer consistent with equilibrium because the generous type would do better to choose $x_{g}$. The unique equilibrium, therefore, gives $s(M)=x_{m}$ and $s(G)=x_{g}<\widehat{x}$. The expected donation is, thus, strictly lower than exact reporting if there was a separating equilibrium and unchanged if there was a trivially separating equilibrium.

\section{Proposition 3 (Low threshold)}

First we characterise the equilibrium, then consider the implications for donations. Fix $\widehat{x}$ such that $x_{m}<\widehat{x} \leq x_{L}$ and let $s(M)=\widehat{x}$ and $s(G)=$ $\max \left\{x_{g}, \widehat{x}\right\}$. Given single-crossing properties 1 and 2, an inference of $q(x)=$ 0 for all $x<\widehat{x}$ is consistent with the intuitive criterion ${ }^{16}$. The miserly type has no incentive to deviate given $\widehat{x} \leq x_{L}$. This implies, given single-crossing property 2 , that neither does the generous type. Thus we have an equilibrium.

We begin to consider the implications for donations by treating the case $\widehat{x}=x_{L}$. Given equilibrium donations, the generous type must donate at least $x_{L}$. If the generous type donates $x_{L}$, then the expected donation is greater than that with exact reporting iff $x_{L}>p x_{H}+(1-p) x_{m}$, or,

$$
\frac{x_{L}-x_{m}}{p E}>\frac{x_{H}-x_{m}}{E} \text {. }
$$

Given real number $\bar{p} \in[0,1]$, define $\bar{x}>x_{m}$ such that $m\left(x_{m}\right)=m(\bar{x})+\bar{p} E$ and $F:=\frac{\bar{x}-x_{m}}{\bar{p} E}$. Note that if $\bar{p}=p$ then $F$ equals the LHS of inequality (2)

\footnotetext{
${ }^{16}$ Indeed, this is the only permissible inference if we apply the D1 criterion instead (Editor/referees: see Appendix 2).
} 
and if $\bar{p}=1$ then $F$ equals the RHS of inequality (2). Further note that,

$$
\begin{aligned}
\frac{d F}{d \bar{p}} & =\frac{\bar{p} E-\left|m^{\prime}(\bar{x})\right|\left(\bar{x}-x_{m}\right)}{\bar{p}^{2} E\left|m^{\prime}(\bar{x})\right|}=\frac{\bar{x}-x_{m}}{\bar{p}^{2} E\left|m^{\prime}(\bar{x})\right|}\left[-\frac{m(\bar{x})-m\left(x_{m}\right)}{\bar{x}-x_{m}}-\left|m^{\prime}(\bar{x})\right|\right], \\
& =\frac{\bar{x}-x_{m}}{\bar{p}^{2} E\left|m^{\prime}(\bar{x})\right|}\left[\left|m^{\prime}\left(x: x_{m}<x<\bar{x}\right)\right|-\left|m^{\prime}(\bar{x})\right|\right],
\end{aligned}
$$

where the final step uses the mean value theorem. Concavity of $m$ implies that $\left|m^{\prime}\left(x: x_{m}<x<\bar{x}\right)\right|<\left|m^{\prime}(\bar{x})\right|$. Thus $d F / d \bar{p}<0$ and $x_{L}>p x_{H}+$ $(1-p) x_{m}$. Thus a threshold of $x_{L}$ gives a strictly higher expected donation.

All that remains is to consider what happens for lower thresholds. It is clear that the expected donation is monotonically decreasing in $\widehat{x}$ and so we need to find the point below which donations are no higher than with exact reporting. With the threshold of $\widehat{x}$ the expected donation is $p \max \left\{x_{g}, \widehat{x}\right\}+$ $(1-p) \widehat{x}$. With exact reporting the expected donation is $p \max \left\{x_{g}, x_{H}\right\}+$ $(1-p) x_{m}$. This explains the stated condition $\widehat{x}>x_{L L}$.

\section{Proposition 4 (Low category pooling)}

Suppose $\widehat{x} \in\left[x_{g L}, x_{g H}\right]$ and let $s(M)=x_{m}$ and $s(G)=x_{g}$, implying $q(x)=p$ for all $x<\widehat{x}$. Given that $\widehat{x} \geq x_{g L}$, not deviating is a best-response for any $q(\widehat{x})$ for the generous type and single-crossing property 2 implies the same for the miserly type. The implication for the expected donation follows immediately.

\section{Appendix 2: Category reporting equilibria char- acterisation}

This appendix (not for publication) gives a complete charaterisation of all the equilibria of our category reporting model where mixed strategies are permitted and $x_{m}<\widehat{x} \leq x_{g H}$. Let $\sigma(x, T)$ denote the probability a player of type $T$ donates $x$. We take it as given that $\sigma$ has finite support. Where the intuitive criterion is not sufficiently strong, we refine our signalling equilibria with the D1 criterion instead.

Proposition A1 (High threshold): If there is a high threshold of $\max \left\{x_{H}, x_{g}\right\}<$ $\widehat{x} \leq x_{g H}$, then the miserly type donates $x_{m}$ and there exists a,

(a) Separating equilibrium where a generous type donates $\widehat{x}$, 
(b) Hybrid equilibrium where a generous type donates $x_{g}$ with probability $\frac{1-p}{p}\left[\frac{E}{g\left(x_{g}\right)-g(\hat{x})}-1\right]$ and $\widehat{x}$ with probability $\frac{1}{p}\left[1-\frac{(1-p) E}{g\left(x_{g}\right)-g(\hat{x})}\right]$, if $x_{g L}<\hat{x}<$ $x_{g H}$,

(c) Pooling equilibrium where a generous type donates $x_{g}$ if $\hat{x} \geq x_{g L}$.

Proof: Note by single-crossing property 2 and the definition of $x_{H}, x_{g H}>$ $x_{H}$. Given $\widehat{x}>\max \left\{x_{H}, x_{g}\right\}$, a miserly type must donate $x_{m}$. All that remains is to determine a generous type's donation. Given $q(x)=q\left(x^{\prime}\right)$ if $x, x^{\prime}<\widehat{x}$ or $x, x^{\prime} \geq \widehat{x}$, clearly in equilibrium $\sigma(x, G)=0$ for all $x \notin\left\{x_{g}, \widehat{x}\right\}$. Consider each type of equilibrium in turn.

Separating: Standard reasoning establishes existence of an equilibrium where $\sigma(\widehat{x}, G)=1 .^{17}$

Hybrid: Let $\sigma\left(x_{g}, G\right), \sigma(\widehat{x}, G)>0$, implying $g\left(x_{g}\right)-g(\hat{x})=(1-q(x<\widehat{x})) E$. Substituting for $q(x<\widehat{x})$ gives,

$$
\sigma\left(x_{g}, G\right)=\frac{1-p}{p}\left[\frac{E}{g\left(x_{g}\right)-g(\hat{x})}-1\right] .
$$

Note $\sigma\left(x_{g}, G\right)<1$ iff $\hat{x}>x_{g L}$ and $\sigma\left(x_{g}, G\right)>0$ iff $\hat{x}<x_{g H}$.

Pooling: Let $\sigma\left(x_{g}, G\right)=1$, implying inference $q(x<\widehat{x})=p . \quad$ If $\widehat{x} \geq x_{g L}$, $x_{g}$ is a best-response. If $\widehat{x}<x_{g L}$, note that the intuitive criterion requires $q(\widehat{x})=1$ since $\widehat{x}>x_{H}$, then $x_{g}$ is not a best-response. ${ }^{18}$

Proposition A2 (Low threshold): If there is a low threshold of $x_{m}<$ $\widehat{x} \leq x_{L}$ then there exists a

(a) Pooling equilibrium where a generous type donates $\max \left\{x_{g}, \widehat{x}\right\}$ and a miserly type donates $\widehat{x}$,

\footnotetext{
${ }^{17}$ Note that there is no out of equilibrium signal if there is a separating or hybrid PBE. Thus no refinement that works by restricting off path beliefs has any bite. These two are the only separating/hybrid PBE.

${ }^{18}$ Note: Other refinements may give slightly different results on pooling. D1 would also set $q(\hat{x})=1$ as is less costly for generous type to take action $\hat{x}$, so same result as intuitive. Undefeated equilibrium only permits off-path inference when could get higher payoff in another equilibrium, however highest payoff is in pooling, so in this case, perhaps it sustains pooling even if $g\left(x_{g}\right)-g(\hat{x})<(1-p) E$. Thus intuitive criterion excludes pooling based on off-path inference of a low type.
} 
(b) Hybrid equilibrium where a miserly type donates $\widehat{x}$ and a generous type donates $x_{g}<\widehat{x}$ with probability $\frac{1-p}{p}\left[\frac{E}{g\left(x_{g}\right)-g(\widehat{x})}-1\right]$ and $\widehat{x}$ with probability $\frac{1}{p}\left[1-\frac{(1-p) E}{g\left(x_{g}\right)-g(\hat{x})}\right]$ if $\widehat{x}>x_{g L}$,

(c) Pooling equilibrium where a miserly type donates $x_{m}$ and a generous type donates $x_{g}$ if $\widehat{x} \geq x_{g L}$.

Proof: Which is greater of $x_{g}$ and $\widehat{x}$ is unclear, thus consider each case separately.

Case 1: $\widehat{x} \leq x_{g}$.

In equilibrium, $\sigma(x, G)=0$ for all $x \notin\left\{\widehat{x}-\varepsilon, x_{g}\right\}$ and $\sigma(x, M)=0$ for all $x \notin\left\{x_{m}, \widehat{x}\right\}$. Consider each type of equilibrium in turn.

Separating: If $\sigma(\widehat{x}, M)=\sigma(\widehat{x}-\varepsilon, G)=1$ a miserly type deviates to $x_{m}$, increasing esteem and intrinsic utility. If $\sigma\left(x_{m}, M\right)=\sigma\left(x_{g}, G\right)=1$, a miserly type deviates to $\widehat{x}$ given $\widehat{x}<x_{H}$. Therefore separating equilibria do not exist.

Hybrid: If $\sigma\left(x_{m}, M\right), \sigma(\widehat{x}, M), \sigma(\widehat{x}-\varepsilon, G), \sigma\left(x_{g}, G\right)>0$ then $g\left(x_{g}\right)-$ $g(\widehat{x}-\varepsilon)=[q(x<\widehat{x})-q(\widehat{x})] E$ and $m(\widehat{x})-m\left(x_{m}\right)=[q(x<\widehat{x})-q(\widehat{x})] E$, a contradiction given $g\left(x_{g}\right)-g(\widehat{x}-\varepsilon)>0$ and $m(\widehat{x})-m\left(x_{m}\right)<0$. Consider potential hybrid equilibria where only one type mixes. Where $\sigma\left(x_{m}, M\right)=1$, $\sigma(\widehat{x}, M)=1$ or $\sigma(\widehat{x}, G)=1$ one type can deviate to increase both intrinsic and esteem utility. This leaves $\sigma\left(x_{g}, G\right)=1$ and a miserly mixing. Then $m(\widehat{x})-m\left(x_{m}\right)=[q(x<\widehat{x})-q(\widehat{x})] E$ which gives,

$$
\sigma(\widehat{x}, M)=\frac{p}{(1-p)}\left[\frac{E}{m\left(x_{m}\right)-m(\widehat{x})}-1\right] .
$$

Note $\sigma(\widehat{x}, m) \geq 1$ given $\widehat{x} \leq x_{L}$. Therefore no hybrid equilibria exist.

Pooling: If $\sigma\left(x_{m}, M\right)=\sigma(\widehat{x}-\varepsilon, G)=1$, the D1 criterion requires $q(\widehat{x})=1$, but then a generous type deviates ${ }^{19}$. If $\sigma(\widehat{x}, M)=\sigma\left(x_{g}, G\right)=1$, the D1 criterion requires $q(x<\widehat{x})=0$, then a generous type does best to donate $x_{g}$ and a miserly type $\widehat{x}$ given $\widehat{x} \leq x_{L}$. Therefore there exists a pooling equilibrium.

\footnotetext{
${ }^{19}$ Note that this equilibrium may be supported by the intuitive criterion as it would permit $q(\widehat{x})$ to be low. The intuitive criterion would give similarly unintuitive results in other pooling equilibria too.
} 
Case 2: $\widehat{x}>x_{g}$

In equilibrium, $\sigma(x, G)=0$ for all $x \notin\left\{x_{g}, \widehat{x}\right\}$ and $\sigma(x, M)=0$ for all $x \notin\left\{x_{m}, \widehat{x}\right\}$. Consider each type of equilibrium in turn.

Separating: If $\sigma(\widehat{x}, M)=\sigma\left(x_{g}, G\right)=1$ a miserly type deviates to $x_{m}$, increasing esteem and intrinsic utility. If $\sigma\left(x_{m}, M\right)=\sigma(\widehat{x}, G)=1$ a miserly type deviates to $\widehat{x}$ as $\widehat{x}<x_{H}$. Therefore separating equilibria do not exist.

Hybrid: If $\sigma\left(x_{m}, M\right), \sigma(\widehat{x}, M), \sigma\left(x_{g}, G\right), \sigma(\widehat{x}, G)>0$ then $m\left(x_{m}\right)-m(\widehat{x})=$ $[q(\widehat{x})-q(x<\widehat{x})] E$ and $g\left(x_{g}\right)-g(\widehat{x})=[q(\widehat{x})-q(x<\widehat{x})] E$, contradicting single-property 2 which implies $m\left(x_{m}\right)-m(\widehat{x})>g\left(x_{g}\right)-g(\widehat{x})$. Consider potential hybrid equilibrium with only one type mixing. Where $\sigma(\widehat{x}, M)=1$ or $\sigma\left(x_{g}, G\right)=1$ a miserly type can deviate to increase instrinsic and esteem utility. If $\sigma(\widehat{x}, g)=1$ and a miserly type mixes implying $m\left(x_{m}\right)=m(\widehat{x})+$ $q(\widehat{x}) E$ we have

$$
\sigma(\widehat{x}, M)=\frac{p}{1-p}\left[\frac{E}{m\left(x_{m}\right)-m(\widehat{x})}-1\right] .
$$

Note that $\sigma(\widehat{x}, M) \geq 1$ given $\widehat{x} \leq x_{L}$. Finally, if $\sigma\left(x_{m}, M\right)=1$ and a generous type mixes implying $g\left(x_{g}\right)+q(x<\widehat{x}) E=g(\widehat{x})+E$ we have

$$
\sigma\left(x_{g}, G\right)=\frac{1-p}{p}\left[\frac{E}{g\left(x_{g}\right)-g(\widehat{x})}-1\right] .
$$

Note that $\sigma\left(x_{g}, G\right)>0$ given $\widehat{x}<x_{H}$ and $\sigma\left(x_{g}, G\right)<1$ iff $\widehat{x}>x_{g L}$. Therefore, there exists a hybrid equilibrium iff $\widehat{x}>x_{g L}$.

Pooling: If $\sigma\left(x_{m}, m\right)=\sigma\left(x_{g}, g\right)=1$ the D1 criterion requires $q(\widehat{x})=1$, a generous type deviates iff $\widehat{x}<x_{g L}$. If $\sigma(\widehat{x}, m)=\sigma(\widehat{x}, g)=1$, the D1 criterion requires $q(x<\widehat{x})=0$. Given $\widehat{x} \leq x_{L}, \widehat{x}$ is a best response for a miserly type, further single crossing property 2 implies $\widehat{x}$ is also a bestresponse for a generous type. Therefore there exists one pooling equilibrium if $\widehat{x}<x_{g L}$, otherwise there are two.

Proposition A3 (Intermediate threshold): If there is an intermediate threshold of $x_{L}<\widehat{x} \leq \max \left\{x_{g}, x_{H}\right\}$ then there exists a:

(a) Separating equilibrium where a miserly type donates $x_{m}$ and a generous type donates $\max \left\{x_{g}, \widehat{x}\right\}$ if $x_{H} \leq \widehat{x} \leq x_{g}$ or $\widehat{x}=x_{H}>x_{g}$, 
(b) Hybrid equilibrium where a generous type donates $\max \left\{x_{g}, \widehat{x}\right\}$ and a miserly type donates $\widehat{x}$ with probability $\frac{p}{1-p}\left[\frac{E}{m\left(x_{m}\right)-m(\widehat{x})}-1\right]$ and $x_{m}$ with probability $\frac{1}{1-p}\left[1-\frac{p E}{m\left(x_{m}\right)-m(\widehat{x})}\right]$ if $\widehat{x}<x_{H}$,

(c) Hybrid equilibrium where a miserly type donates $x_{m}$ and a generous type donates $x_{g}$ with probability $\frac{1-p}{p}\left[\frac{E}{g\left(x_{g}\right)-g(\widehat{x})}-1\right]$ and $\widehat{x}$ with probability $\frac{1}{p}\left[1-\frac{(1-p) E}{g\left(x_{g}\right)-g(\hat{x})}\right]$ if $x_{g}<\widehat{x}<x_{g L}$,

(d) Pooling equilibrium where a miserly type donates $x_{m}$ and a generous type donates $x_{g}$ if $\widehat{x} \geq x_{g L}$.

Proof: It is unclear whether $x_{g}$ is greater than $\widehat{x}$, thus consider each case.

Case 1: $\widehat{x} \leq x_{g}$

In equilibrium, $\sigma(x, G)=0$ for all $x \notin\left\{\widehat{x}-\varepsilon, x_{g}\right\}$ and $\sigma(x, M)=0$ for all $x \notin\left\{x_{m}, \widehat{x}\right\}$. Consider each type of equilibrium in turn.

Separating: If $\sigma\left(x_{m}, M\right)=\sigma\left(x_{g}, G\right)=1$ a miserly type does best to donate $x_{m}$ iff $\widehat{x} \geq x_{H}$. If $\sigma(\widehat{x}, M)=\sigma(\widehat{x}-\varepsilon, G)=1$ a miserly type increases intrinsic and esteem utility by deviating to $x_{g}$. Therefore a separating equilibrium exists if $\widehat{x} \geq x_{H}$.

Hybrid: For $\sigma\left(x_{m}, M\right), \sigma(\widehat{x}, M), \sigma\left(x_{g}, G\right), \sigma(\widehat{x}-\varepsilon, G)>0$ we need $m\left(x_{m}\right)-$ $m(\widehat{x})=[q(\widehat{x})-q(x<\widehat{x})] E$ and $g(\widehat{x}-\varepsilon)-g\left(x_{g}\right)=[q(\widehat{x})-q(x<\widehat{x})] E$, a contradiction since $m\left(x_{m}\right)-m(\widehat{x})<0$ and $g(\widehat{x}-\varepsilon)-g\left(x_{g}\right)>0$. Now consider potential hybrid equilibria where only one type mixes. If $\sigma\left(x_{m}, M\right)=1$, $\sigma(\widehat{x}, M)=1$ or $\sigma(\widehat{x}-\varepsilon, G)=1$ then one type can increase esteem and intrinsic utility by deviating. This leaves $\sigma\left(x_{g}, G\right)=1$ and a miserly type mixes implying $m\left(x_{m}\right)=m(\widehat{x})+q(\widehat{x}) E$ giving,

$$
\sigma(\widehat{x}, M)=\frac{p}{1-p}\left[\frac{E}{m\left(x_{m}\right)-m(\widehat{x})}-1\right] .
$$

Note $\sigma(\widehat{x}, M)>0$ iff $\widehat{x}<x_{H}$ and $\sigma(\widehat{x}, M)<1$ given $\widehat{x}>x_{L}$. Therefore if $\widehat{x}<x_{H}$ a hybrid equilibrium exists.

Pooling: If $\sigma\left(x_{m}, M\right)=\sigma(\widehat{x}-\varepsilon, G)=1$ the D1 criterion implies $q(\widehat{x})=1$ and a generous type deviates. If $\sigma(\widehat{x}, M)=\sigma\left(x_{g}, G\right)=1$ then a miserly type deviates since $\widehat{x}>x_{L}$. There exist no pooling equilibria. 
Case 2: $\widehat{x}>x_{g}$ (implying $x_{H}>x_{g}$ )

In equilibrium, $\sigma(x, G)=0$ for all $x \notin\left\{\widehat{x}, x_{g}\right\}$ and $\sigma(x, M)=0$ for all $x \notin\left\{x_{m}, \widehat{x}\right\}$. Consider each type of equilibrium in turn.

Separating: If $\sigma\left(x_{m}, M\right)=\sigma(\widehat{x}, G)=1$ the miserly type does best to donate $x_{m}$ iff $\widehat{x}=x_{H}$ and a generous type does best to donate $\widehat{x}$ given $\widehat{x}=x_{H}$ and single-crossing property 2. Thus an equilibrium exists. If $\sigma(\widehat{x}, M)=$ $\sigma\left(x_{g}, G\right)=1$ a miserly type deviates. There exists a separating equilibrium if $\widehat{x}=x_{H}$.

Hybrid: If $\sigma\left(x_{m}, M\right), \sigma(\widehat{x}, M), \sigma\left(x_{g}, G\right), \sigma(\widehat{x}, G)>0$ then $m\left(x_{m}\right)-m(\widehat{x})=$ $[q(\widehat{x})-q(x<\widehat{x})] E$ and $g\left(x_{g}\right)-g(\widehat{x})=[q(\widehat{x})-q(x<\widehat{x})] E$, a contradiction since single-crossing property 2 implies $m\left(x_{m}\right)-m(\widehat{x})>g\left(x_{g}\right)-g(\widehat{x})$. Consider potential hybrid equilibria with only one type mixing. If $\sigma\left(x_{g}, G\right)=$ 1 or $\sigma(\widehat{x}, M)=1$ then one type can increase esteem and intrinsic utility by deviating. Suppose $\sigma(\widehat{x}, G)=1$ and the miserly type mixes implying $m\left(x_{m}, m\right)=m(\widehat{x}, m)+q(\widehat{x}) E$ thus,

$$
\sigma(\widehat{x}, M)=\frac{p}{1-p}\left[\frac{E}{m\left(x_{m}\right)-m(\widehat{x})}-1\right] .
$$

Note $\sigma(\widehat{x}, M)<1$ given $\widehat{x}>x_{L}$ and $\sigma(\widehat{x}, M)>0$ iff $\widehat{x}<x_{H}$. Given $\sigma(\widehat{x}, M)$, a generous type has no incentive to deviate if $g\left(x_{q}\right)-g(\widehat{x})<$ $m\left(x_{m}\right)-m(\widehat{x})$, which holds by single-crossing property 2 . Therefore we have an equilibrium. Finally, suppose $\sigma\left(x_{m}, M\right)=1$ and a generous type mixes implying $g\left(x_{g}\right)-g(\widehat{x})=[1-q(x<\widehat{x})] E$, giving,

$$
\sigma\left(x_{g}, G\right)=\frac{1-p}{p}\left[\frac{E}{g\left(x_{g}\right)-g(\widehat{x})}-1\right] .
$$

Note $\sigma\left(x_{g}, g\right)>0$ given $\widehat{x} \leq x_{H}$ and single-crossing property 2 and $\sigma\left(x_{g}, G\right)<$ 1 iff $\widehat{x}<x_{g L}$. Given $\sigma\left(x_{g}, G\right)$ the miserly type has no incentive to deviate if $m\left(x_{m}\right)-m(\widehat{x})>g\left(x_{g}\right)-g(\widehat{x})$, which holds by single crossing property 2 . Therefore there are two hybrid equilibria: One existing if $x_{g}<\widehat{x}<x_{H}$, the second existing if $x_{g}<\widehat{x}<x_{g L}$.

Pooling: Given $\widehat{x}>x_{L}$, a miserly type deviates to $x_{m}$ when $\sigma(\widehat{x}, M)=$ $\sigma(\widehat{x}, G)=1$. When $\sigma\left(x_{m}, M\right)=\sigma\left(x_{g}, G\right)=1$ the D1 criterion implies $q(\widehat{x})=1, x_{g}$ is a best-response for the generous type iff $\widehat{x} \geq x_{g L}$. Therefore there exists a pooling equilibrium if $\widehat{x} \geq x_{g L}$. 


\section{References}

[1] Alpizar, F., Carlsson, F. and Johansson-Stenman, O. (2008) "Anonymity, reciprocity and conformity: evidence from voluntary contributions to a national park in Costa Rica," Journal of Public Economics, 92: 1047-1060.

[2] Andreoni, J. and Bernheim, B. D. (2009) "Social image and the 5050 norm: A theoretical and experimental analysis of audience effects, Econometrica, 77(5): 1607-1636.

[3] Andreoni, J. and Petrie, R. (2004) "Public goods experiments without confidentiality: a glimpse into fund-raising," Journal of Public Economics, 88: 1605-1623.

[4] Ariely, D., Bracha, A. and Meier, S. (2009) "Doing good or doing well? Image motivation and monetary incentives in behaving procially," American Economic Review, 99(1): 544-555.

[5] Baik, K. H. (2008) "Contests with group specific public-goods prizes," Social Choice and Welfare 30: 103-117.

[6] Barbieri, S. and Malueg, D. A. (2011) "Increasing fundraising success by decreasing donor choice," mimeo.

[7] Battigali, P. and Dufwenberg, M. (2009) "Dynamic psychological games," Journal of Economic Theory 144(1): 1-35.

[8] Bénabou, R. and Tirole, J. (2006) "Incentives and Prosocial Behavior," American Economic Review 96: 1652-1678.

[9] Breeze, B. (2009) "The Coutts Million Pounds Donors Report", available at http://www.coutts.com/philanthropy.

[10] Carpenter, J. and Myers, C. K. (2010) "Why volunteer? Evidence on the role of altruism, image and incentive," Journal of Public Economics 94(11-12): 911-920.

[11] Cartwright, E., and A. Patel (2010) "Public goods, social norms, and naive beliefs" Journal of Public Economic Theory 12: 199-223. 
[12] Che, Y-K. and Gale, I. L. (1998) "Caps on political lobbying," American Economic Review 88(3): 643-651.

[13] Cho, I. and Kreps, D. (1987) "Signalling games and stable equilibria," Quarterly Journal of Economics 102: 179-221.

[14] Daughety, A. F. and Reinganum, J. F. (2010) "Public goods, social pressure and the choice between privacy and publicity," American Economic Journal: Microeconomics 2(2): 191-221.

[15] Drazen, A., Limão, N. and Stratmann, T. (2007) "Political contribution caps and lobby formation: theory and evidence," Journal of Public Economics 91: 723-754.

[16] Ellingsen, T. and Johannesson, M. (2008) "Pride and prejudice: The human side of incentive theory," American Economic Review 98(3): 9901008 .

[17] Ellingsen, T. and Johannesson, M. (2011) "Conspicuous generosity," Journal of Public Economics 95(9-10): 1131-1143.

[18] Feltovich, N., Harbaugh, R. and To, T. (2002) "Too cool for school? Signalling and countersignalling," RAND Journal of Economics 33(4): 630-649.

[19] Fudenberg, D. and Tirole, J. (1996) Game Theory MIT Press.

[20] Frank, R. (1985) Choosing the right pond Oxford University Press.

[21] Gavious, A., Moldovanu, B. and Sela A. (2002) "Bid costs and endogenous bid caps," RAND Journal of Economics 33(4): 709-722.

[22] Glazer, A. and Konrad, K. A. (1996) "A signaling explanation for charity," American Economic Review 86(4): 1019-1028.

[23] Harbaugh, R. and To, T. (2008) "False modesty: When disclosing good news looks bad," mimeo.

[24] Harbaugh, W. T. (1998a) "What do donations buy? A model of philanthropy based on prestige and warm glow," Journal of Public Economics 67: 269-284. 
[25] Harbaugh, W. T. (1998b) "The prestige motive for making charitable transfers," American Economic Review 88(2): 277-282.

[26] Lacetera, N. and Macis, M. (2010) "Social image concerns and prosocial behavior: Field evidence from a nonlinear incentive scheme," Journal of Economic Behavior and Organization 76(2): 225-237.

[27] List, J. A. and Lucking-Reiley, D. (2002) "The effects of seed money and refunds on charitable giving: Experimental evidence from a university capital campaign," Journal of Political Economy 110(1): 215-233.

[28] Mailath, G., M. Okuno-Fujiwara, and A. Postlewaite (1993) "Beliefbased refinements in signalling games", Journal of Economic Theory 60: $241-276$.

[29] McCardle, K. F., Rajaram, K. and Tang, C.S (2009) "A decision analysis tool for evaluating fundraising tiers," Decision Analysis 6(1): 4-13.

[30] Millet, K. and Dewitte, S. (2007) "Altruistic behavior as a costly signal of general intelligence," Journal of Research in Personality 41: 316-326.

[31] Moldovanu, B., Sela, A. and Shi, X. (2007) "Contests for status," Journal of Political Economy 115(2): 338-363.

[32] de Oliveira, A. C. M., Croson, R. T. A. and Eckel, C. (2011) "The giving type: Identifying donors," Journal of Public Economics 95: 428-435.

[33] Riley, J. (2001) "Silver Signals: Twenty-Five Years of Screening and Signaling," Journal of Economic Literature 39: 432-478.

[34] Riley, J. (1979a) "Noncooperative equilibrium and market signalling", American Economic Review 69: 303-307.

[35] Riley, J. (1979b) "Informational equilibrium", Econometrica 47: 331359.

[36] Sieg, H. and Zhang, J. (2011) "The effectiveness of private benefits in Fundraising," International Economic Review, forthcoming.

[37] Sobel, J. (2009) "Signaling games," in Sotomayer (Ed.) Encyclopedia of Complexity and Systems Science Springer. 
[38] Soetevent, A. R. (2005) "Anonymity in donating in a natural context - a field experiment in 30 churches," Journal of Public Economics 89: 2301-2323.

[39] Soetevent, A. R. (2011) "Payment choice, image motivation and contributions to charity: Evidence from a field experiment," American Economic Journal: Economic Policy 3(1): 180-205. 